\title{
Characterization and regulation mechanism analysis of ubiquitin-conjugating family genes in strawberry reveals a potential role in fruit ripening
}

Mengyao $\mathrm{Li}^{1 \dagger}$, Liangxin Wang ${ }^{1 \dagger}$, Yiting Liu ${ }^{1 \dagger}$, Yuanxiu Lin ${ }^{1,2}$, Yunting Zhang ${ }^{1,2}$, Yu Long ${ }^{1}$, Chuanying Luo ${ }^{1}$, Yong Zhang ${ }^{1}$, Qing Chen ${ }^{1}$, Pinwen Chen ${ }^{3}$, Yan Wang ${ }^{1,2}$, Xiaorong Wang ${ }^{1,2}$, Haoru Tang ${ }^{1,2}$ and Ya Luo ${ }^{1^{*}}$

\begin{abstract}
Background: E2 ubiquitin-conjugating (UBC) enzymes are an integral component of the ubiquitin proteasome system that play an important role in plant development, growth, and external stress responses. Several UBC genes have been identified in various plants. However, no studies exploring the functions of UBC genes in regulating fruit of strawberry have been reported. In the present study, a systematic analysis of the entire UBC family members were conducted in the genome of strawberry (Fragaria $\times$ ananassa) based on bioinformatics method, and the gene functioning in strawberry ripening was explored.

Results: A total of 191 UBC genes were identified in the genome of cultivated strawberry. These genes were unevenly distributed across the 28 chromosomes from the 4 subgenomes of cultivated strawberry, ranging from 3 to 11 genes per chromosome. Moreover, the expansion of FaUBC genes in strawberry was mainly driven by WGD. All the FaUBC genes were clarified into 13 groups and most of them were included in the group VI. The gene structure analysis showed that the number of exons varied from 1 to 23, and the structure of genes had few differences within the same groups but a distinction in different groups. Identification of the cis-acting elements of the promoter revealed multiple regulatory elements that responded to plant growth and development, phytohormone responsive, and abiotic and biotic stress. Data from functional annotation indicated that FaUBC genes play a role in a variety of biological processes. The RNA-seq data showed that FaUBC genes displayed different expression pattern during the fruit ripening process and clarified into 6 clusters. In particular, cluster 3 exhibiting a sudden expression increase in the turning red stage were speculated to be involved in fruit ripening. Hence, two FaUBC genes (FaUBC76 and FaUBC78) were selected for gene function analysis by transient over-expression method. The results indicated that FaUBC76 has a positive effect on the fruit development and ripening in strawberry by up-regulating accumulation of anthocyanins. Moreover, expression of some maturity-related genes were also significantly increased, further supporting a role for FaUBC76 in the regulation of fruit ripening or softening. On the contrary, the overexpression of FaUBC78 significantly
\end{abstract}

*Correspondence: luoya945@163.com

${ }^{\dagger}$ Mengyao Li, Liangxin Wang and Yiting Liu contributed equally to this

work.

${ }^{1}$ College of Horticulture, Sichuan Agricultural University,

Chengdu 611130, China

Full list of author information is available at the end of the article

(c) The Author(s) 2022. Open Access This article is licensed under a Creative Commons Attribution 4.0 International License, which permits use, sharing, adaptation, distribution and reproduction in any medium or format, as long as you give appropriate credit to the original author(s) and the source, provide a link to the Creative Commons licence, and indicate if changes were made. The images or other third party material in this article are included in the article's Creative Commons licence, unless indicated otherwise in a credit line to the material. If material is not included in the article's Creative Commons licence and your intended use is not permitted by statutory regulation or exceeds the permitted use, you will need to obtain permission directly from the copyright holder. To view a copy of this licence, visit http://creativecommons.org/licenses/by/4.0/. The Creative Commons Public Domain Dedication waiver (http://creativeco mmons.org/publicdomain/zero/1.0/) applies to the data made available in this article, unless otherwise stated in a credit line to the data. 
increased the firmness of strawberry fruit, indicating that FaUBC78 had a positive role in inhibiting the decrease of strawberry fruit firmness.

Conclusion: Our study not only provide comprehensive information on system evolution and function on UBC genes, but also give a new insight into explore the roles of FaUBC genes in the regulation of strawberry ripening.

Keywords: Strawberry, Ubiquitin-conjugating enzymes, Phylogenetic analysis, Expression patterns analysis, Fruit ripening

\section{Introduction}

As a ubiquitous post-translational modification for eukaryotes, ubiquitination is involved in many cellular processes, including hormone signaling transduction [1], apoptosis [2], and biotic and abiotic stresses stresses [3]. Protein ubiquitination requires the concerted action of ubiquitin-activating enzyme (E1), ubiquitin-conjugating enzyme (E2) and ubiquitin ligase (E3). In the initial step, the E1 transfers ubiquitin (Ub) to the active site cysteine of E2 in an ATP-dependent manner and forms an E2-Ub intermediate. Subsequently, the E2-Ub conjugate interacts with an E3 to ligate $\mathrm{Ub}$ to a lysine side chain of the target protein and build mono- or poly-ubiquitin chains, then the target protein is degraded or modified to perform different functions [4].

The E2 genes exist as a multi-gene family in higher plants, which have been identified in rice [5], tomato [6], banana [7] and potato [8]. Being a key enzyme of ubiquitination process, E2 protein has a conserved UBC domain containing 150-200 amino acids in length [9]. Ubiquitinconjugating enzyme plays a crucial role in plant growth and development [6], and also participates in environmental stresses [10,11], immune response [12, 13], DNA damage and repair [14]. In Arabidopsis, AtUBC32 is an endoplasmic reticulum-associated degradation (ERAD) component that functions in brassinosteroid-mediated salt stress tolerance [15]; AtUBC2 is implicated in repression of flowering [16]; AtUBC13 was involved in epidermal cell differentiation and iron deficiency responses $[17$, 18]. A tomato UBC13-type homologous protein, FNI3, is involved in the regulation of the immune response [19]. The expression levels of Cucumis melo UBC enzyme $(C m U B C)$ were increased under drought and salt stress in melon [20]. Wheat defense systems against Zymoseptoria tritici can be regulated by Triticum aestivum ubiquitin-conjugating enzymes 4 (TaU4) [21]. The Arabidopsis $U B C 22-$ knockout mutants reduced the length and seed number of siliques, and caused an early arrest of nearly $90 \%$ ovules development [22]. It was reported that the regulator RIN (Ripening-inhibitor) of tomato fruit ripening could directly combine with the E2 promoter region, and the fruit color was changed after silencing the E2 gene [6].
Strawberry is a nutritionally important fruit as well as an ideal model plant for studying non-climacteric fruits. There is a growing recognition that fruit ripening regulation can meet the supply-demand balances of market to some extent. To regulate strawberry fruit development and ripening, previous researchers have mainly focused on abscisic acid (ABA) [23], auxin [24, 25] and sucrose [26]. Genome-wide analysis of the UBC genes in cultivated strawberry would be necessary for strawberry fruit development and ripening research. In this study, we performed a genome-wide analysis of UBC genes in strawberry with a focus on gene structure, evolutionary analysis, and expression abundance. Moreover, the role of E2 genes on strawberry fruit development and ripening were analyzed.

Studies have shown that ABA is a key hormone in the regulation of fruit ripening of strawberry and other non-climacteric fruit. ABA can promote the expression of genes related to strawberry fruit ripening [27]. At the same time, ABA can also promote the expression of other genes related to coloring and softening of non-respiratory climacteric fruits, such as chalcone synthase $(\mathrm{CHI})$ and expansin $(E X P)$ genes [28]. Moreover, the reception and transduction of $\mathrm{ABA}$ signals are regulated by protein ubiquitin modification $[29,30]$. It reported that the $u b c 32$ mutant of E2 protein gene showed an insensitive phenotype to $A B A$ during and after seed germination, and $U B C 32$ was a positive regulator of ABA signal [31]. Current studies have confirmed that $\mathrm{ABA}$ plays an important role in strawberry fruit ripening. $\mathrm{ABA}$ is closely related to ubiquitination, whereas ubiquitin modification plays a role in strawberry fruit ripening has not been reported. In view of this, this study intends to explore the function of E2 in strawberry fruit ripening, in order to enrich the regulatory network of strawberry fruit ripening.

\section{Results}

Genome-wide identification, and characteristics of FaUBC genes in strawberry

Based on the genome searching and domain confirmation, a total of 191 FaUBC genes were identified in the genome of cultivated strawberry. All the identified FaUBC genes were renamed according to the order of their chromosome location (Fig. 1). As the result 


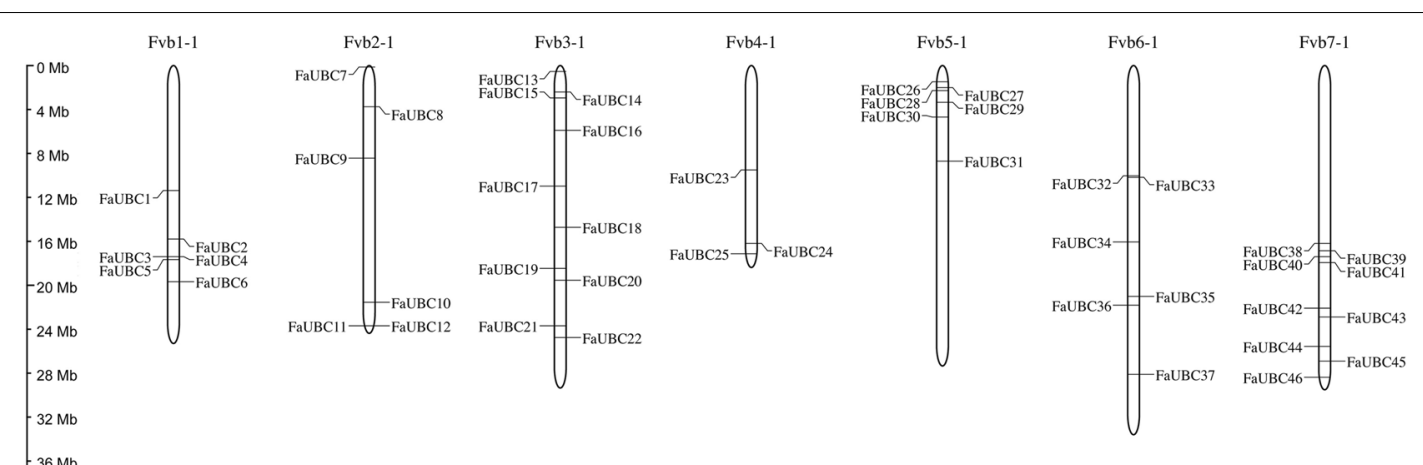

$-30 \mathrm{Mb}$
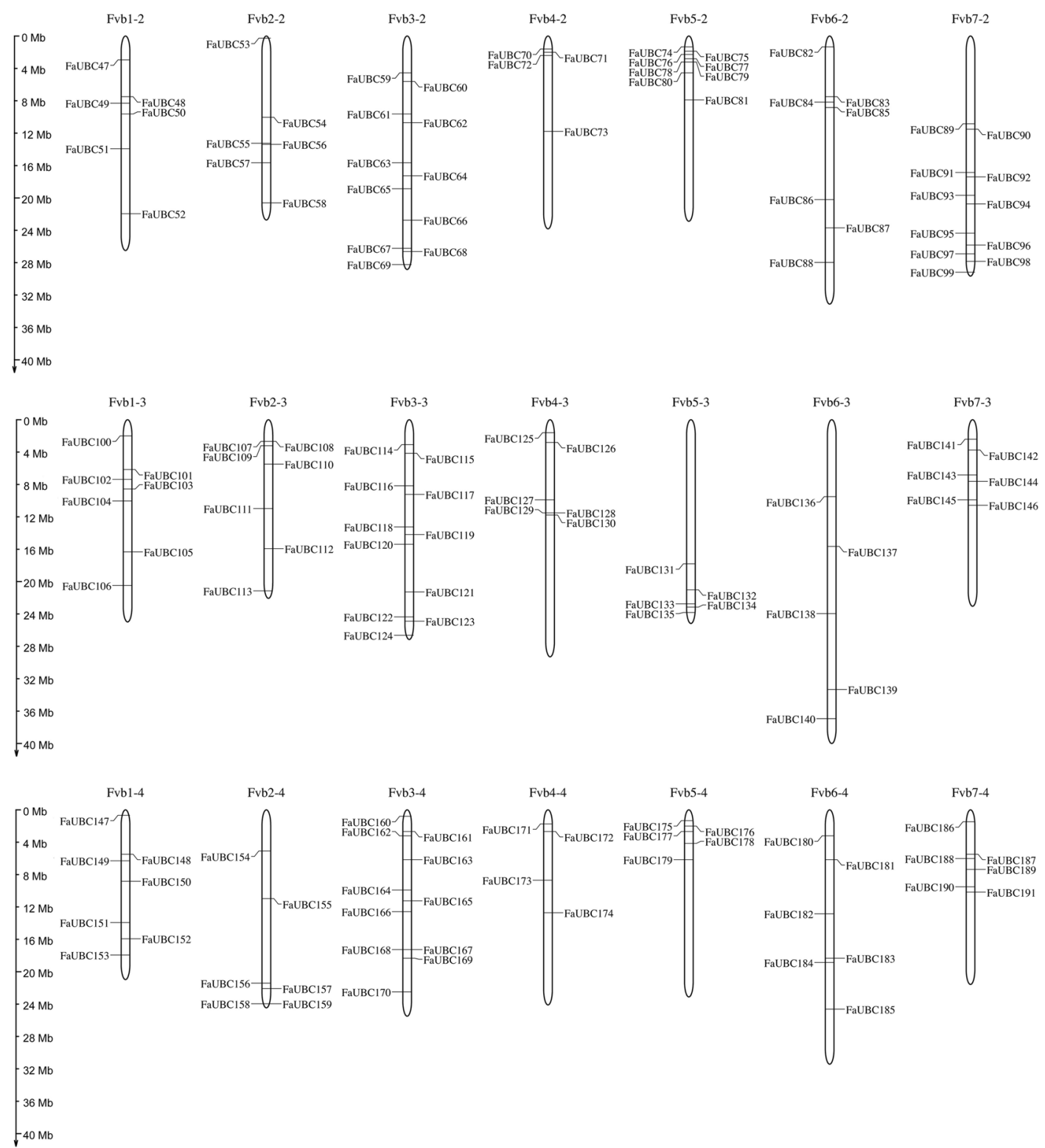

Fig. 1 Chromosomal distribution of UBC genes in the strawberry genome. Chromosome numbers are provided at the top of each chromosome. Scale is in megabases (Mb) 
showed, 191 FaUBC genes were unevenly distributed across the 28 chromosomes in the 4 subgenomes of cultivated strawberry, ranging from 3 to 11 genes per chromosome. A maximum 11 FaUBC genes was located on chromosome 7 and 3 from the second subgenome (Fvb7-2 and Fvb3-2), as well as the chromosome 3 from the third and fourth subgenomes (Fvb3-3 and Fvb3-4), followed by 10 genes on chromosome 3 from the first subgenome (Fvb3-1). On the contrary, the minimum number of FaUBC genes ( 3 members) was distributed on chromosome 4 from the first subgenome (Fvb4-1).

The physicochemical properties of the 191 FaUBC proteins were also analyzed and shown in Table S1. The number of amino acids varied from 67 to 1921, and most of them (145 out of 191, 75.9\%) were from 100 to 300 . The protein molecular weights (MW) were from 7.478 to $216.883 \mathrm{KDa}$, and the isoelectric points (pI) were concentrated from 3.88 to 10.55 . Except for FaUBC134 and FaUBC106, all of the other FaUBC genes didn't contain a signal peptide, which is essential for secreted proteins. Most of the FaUBC genes were predicted to be located in the cytoplasmic, chloroplast and nuclear, also some FaUBC genes were involved in mitochondrial, endoplasmic reticulum, plasma, vacuole, peroxisome and extracellular. Notably, there are some FaUBC genes were predicted to be dual-located, for instance, FaUBC35 and FaUBC139 were located in cytoplasmic and peroxisome, FaUBC125 was located in both nuclear and cytoplasmic, while FaUBC134 was located in extracellular, vacuole or endoplasmic reticulum.

\section{Classification and structural analysis of FaUBC proteins in strawberry}

According to the classification of UBCs in Arabidopsis, all the FaUBC proteins were classified into 13 groups (Fig. 2), most (37 members) of which were included in the group VI, followed by group IV ( 32 members). The gene structure was also investigated (Fig. S1): the number of exons varied from 1 to 23 , and the structure of genes had few differences within the same groups but a distinction in different groups. In addition, the motif analysis showed that 10 motifs were found in the amino acids sequences of FaUBC proteins (Fig. S1). Motif 1 is Ubiquitin-conjugating domain and found in all of the FaUBC sequences. Motif 10 is specific in group XIV, while motif 9 encoding an Ubiquitin-conjugating domain and motif 8 is specific in group XI. The motifs 5 and 2 were also detected as Ubiquitinconjugating domain and found in most of the FaUBC sequences.

\section{Synteny and evolutionary analysis of FaUBC genes in strawberry}

The synteny relationship between Arabidopsis AtUBC genes and strawberry FaUBC genes was investigated to explore the origin and evolutionary process (Fig. 3). A total of 233 pairs of UBC genes were identified as collinear pairs in strawberry, while 78 collinear pairs were identified between Arabidopsis and strawberry (Table S2). Subsequently, the origins of duplication events of FaUBC genes in strawberry were detected using MCScanX package. The result showed that five types of duplication events were detected, including whole genome duplication (WGD) or segmental (WGD/ segmental), Dispersed, Tandem, Singleton and Proximal duplication (Table S1). Most of which were WGD/ segmental, followed by Dispersed with a number of 16 genes. Only FaUBC3 and FaUBC4, FaUBC128 and FaUBC129 were duplicated from Tandem duplication. In addition, FaUBC58 was detected in Singleton duplication event, while FaUBC78, FaUBC79 and FaUBC168 were duplicated from Proximal. These results suggested that the expansion of FaUBC genes in strawberry was mainly driven by WGD. Additionally, the number of non-synonymous substitutions per non-synonymous sites (Ka), the number of synonymous substitutions per synonymous sites (Ks) and the $\mathrm{Ka} / \mathrm{Ks}$ ratio for paralogous gene pairs of FaUBC genes were calculated (Table S3). The results showed that the $\mathrm{Ka}$ and $\mathrm{Ks}$ ranged from 0 to 4.1 and 0.006-3.617 for identified paralogs respectively, the ratio of $\mathrm{Ka} / \mathrm{Ks}$ ranged from 0 to 2.09. Most of the protein-coding genes have a $\mathrm{Ka} / \mathrm{Ks}$ ratio of less than one, and only 23 gene pairs exhibited positive selection with a $\mathrm{Ka} / \mathrm{Ks}$ ratio greater than one.

To illustrate the evolutionary relationship of UBC genes, a collinearity analysis of UBC genes among peach (Prunus persica), strawberry (Fragaria $\times$ ananassa), and pear (Pyrus communis) was constructed. The result revealed 69 and 74 UBC paralogous gene pairs in strawberry/peach and strawberry/pear, respectively (Fig. 4), and the ratio of $\mathrm{Ka} / \mathrm{Ks}$ for each gene pair was analyzed (Table S4). According to the value of Ka and Ks, all the $\mathrm{Ka} / \mathrm{Ks}$ ratios were lower than one, indicating that these paralogous gene pairs were under a strong purifying selection during evolution.

\section{Analysis of the cis-acting elements of the FaUBC genes}

To further predict the function of the FaUBC genes, the cis regulatory elements in the UBC promoter regions were analyzed. The results showed that in addition to the basic core sequences TATA-box and CAAT-box which are unique to higher plant promoters, a total of 4642 elements belong to 76 cis element types were 


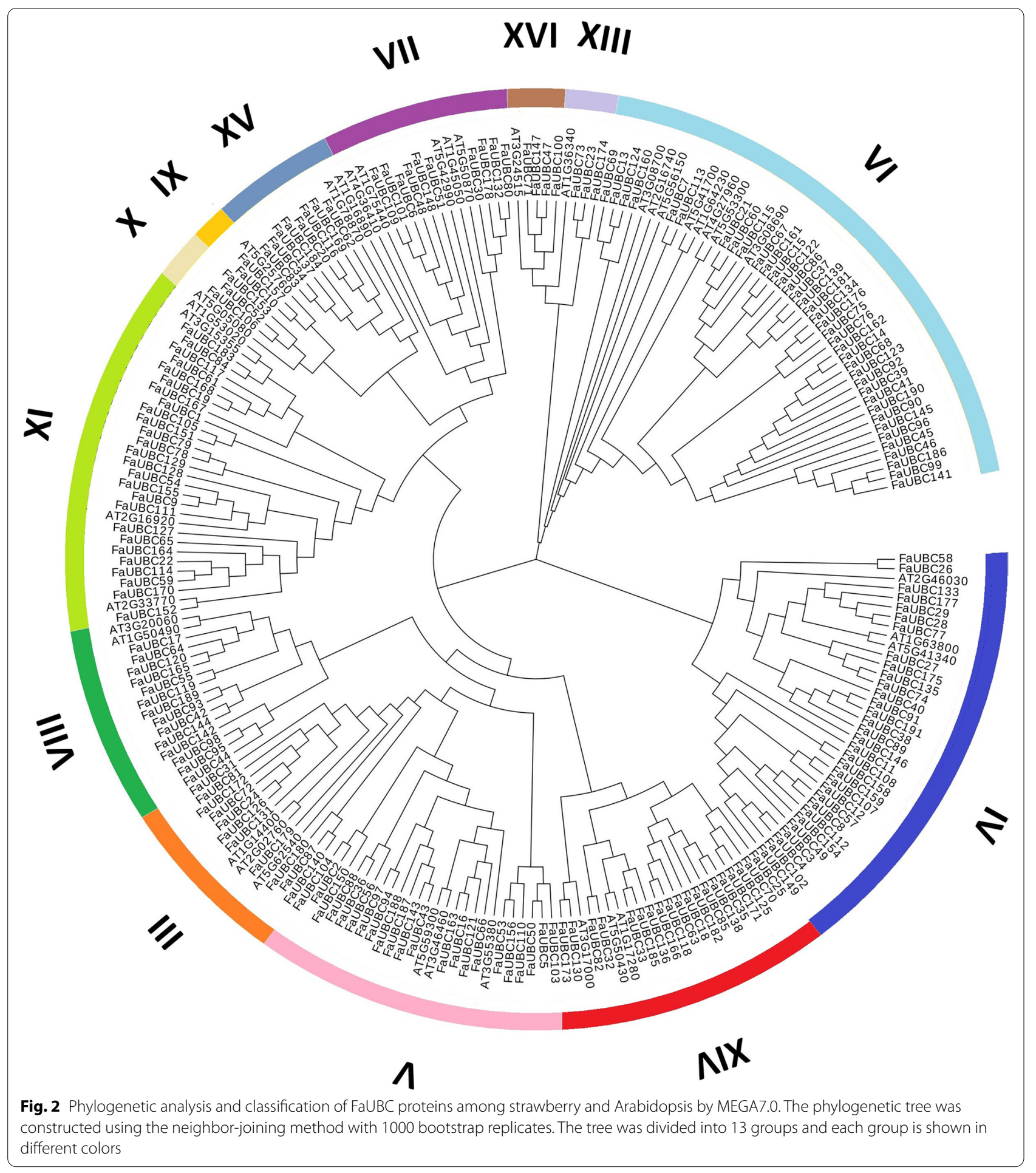

identified in 191 UBC genes (Table S5). Most genes contain 21-30 cis elements, among them, FaUBC176 contains the largest number of elements, with 53 elements, followed by FaUBC88, FaUBC29, FaUBC76, and FaUBC28 (Fig. 5A). The element types contained in each UBC promoter also vary greatly, most of which are in 9-21 types (Fig. 5B). Based on the functional annotation, all the elements were grouped into three major classes: plant growth and development, phytohormone responsive, and abiotic and biotic stress 


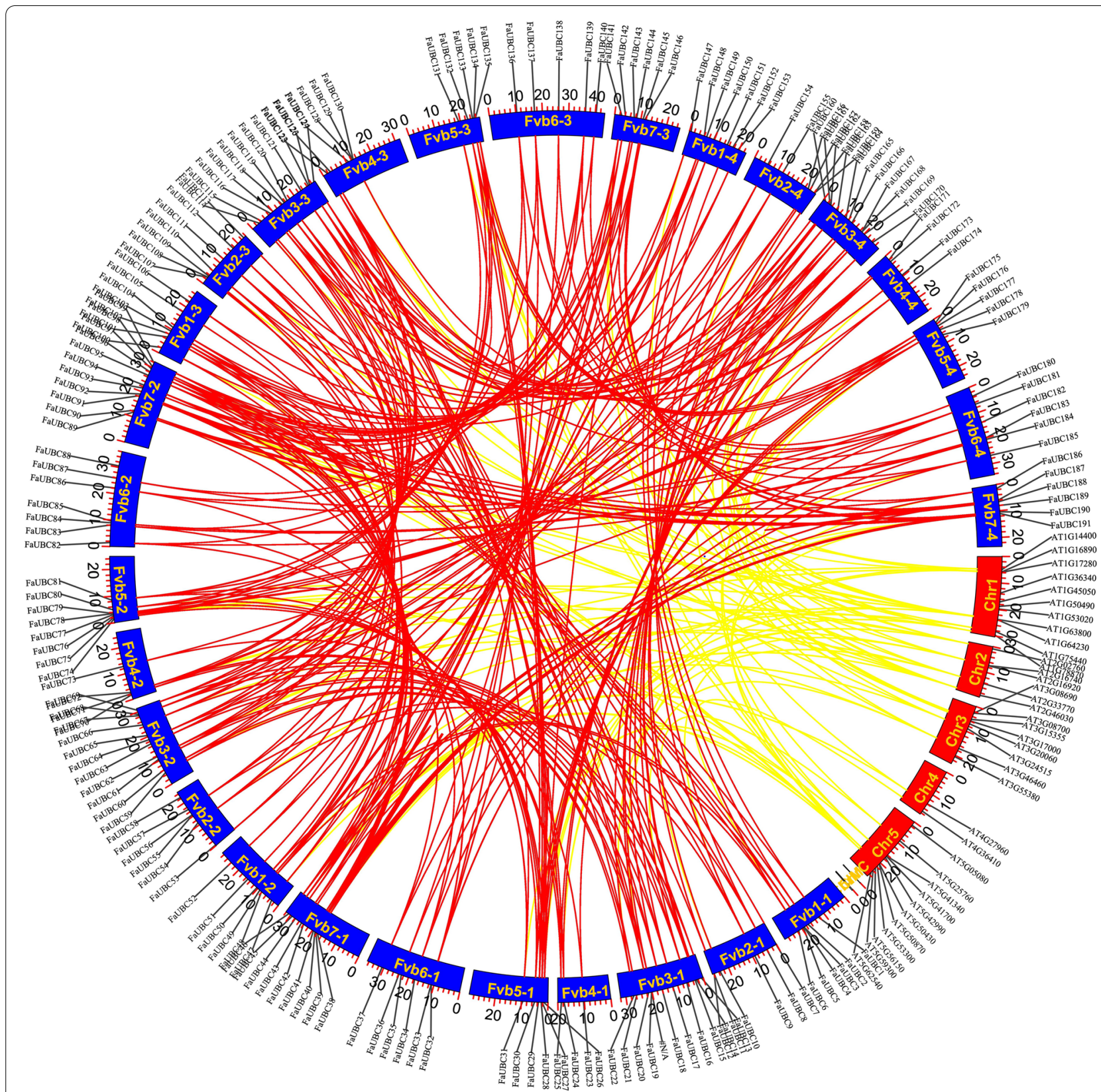

Fig. 3 Synteny analysis of FaUBC genes and AtUBC genes. The chromosomes of strawberry and Arabidopsis were showed as blue and red colors as circular, respectively. The position of UBC genes were marked as black line on the chromosome. Gene pairs in the strawberry/strawberry and strawberry/Arabidopsis with synteny relationships were represented with red and yellow curves, respectively

(Fig. 5C). A total of 126, 93 and 90 FaUBC members were observed to contain G-box, Box 4, and GT1-motif that are involved in light response during plant growth and development, respectively, indicating that they may be important in light response and photosynthetic product accumulation. Among the hormone-related elements, 239 ABA response elements (ABRE), 211 methyl jasmonate response elements (CGTCA-motif), and salicylic acid response elements (TCA-element) were found in multiple UBC genes. In addition, drought response elements (MYB and MYC), anaerobic induction (ARE), and stress response elements (STRE) were also detected in the promoters of multiple UBC genes. 


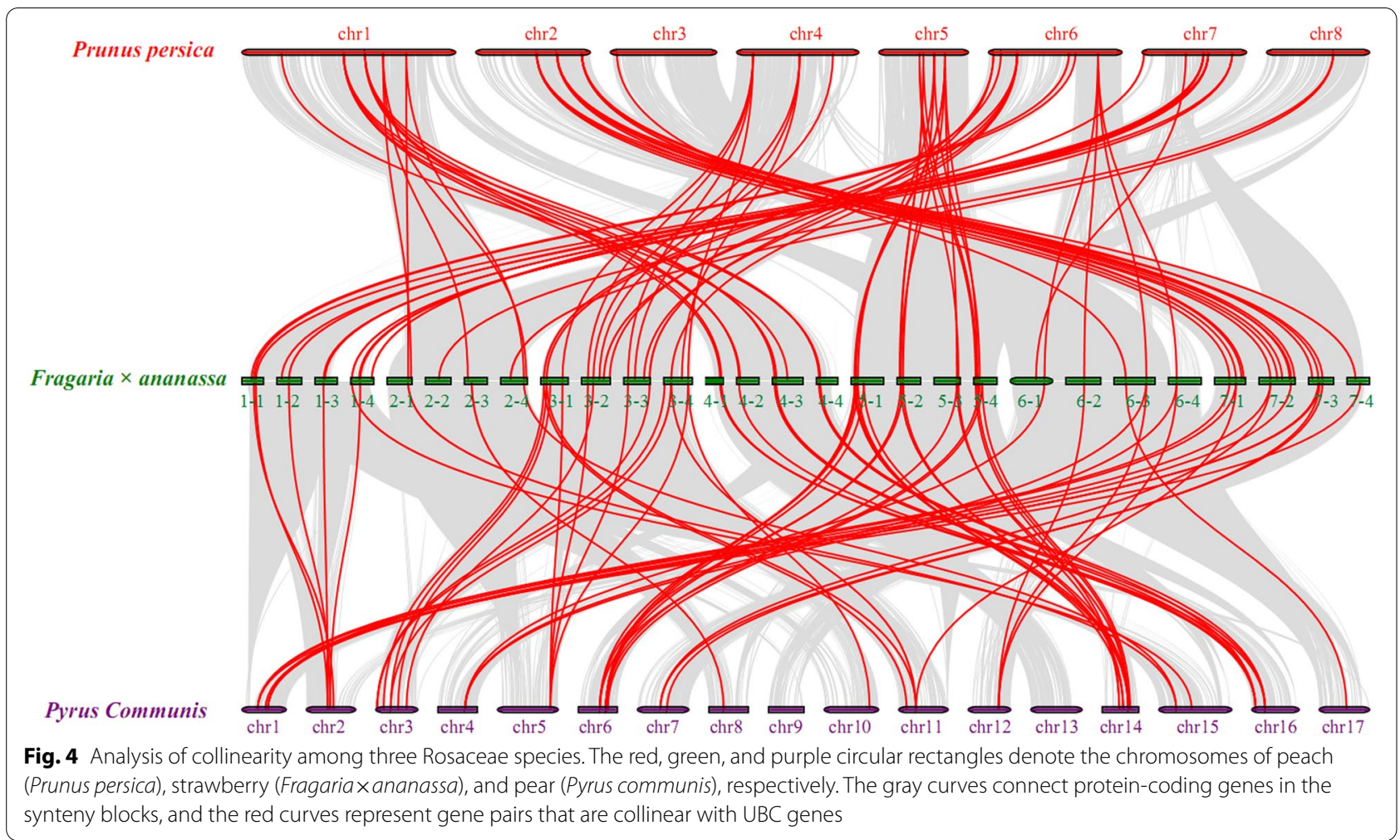

\section{Functional annotation of FaUBC genes}

The GO functional analysis of all FaUBC genes yielded 161 GO function terms ( $p$-value $<0.05)$, including 122 biological process (BP) entries, 10 cell component (CC) entries, and 29 molecular function (MF) entries (Table S6). The GO terms "catalytic activity" and "transferase activity" were described the greatest number of genes with 168 and 167 members, and "protein ubiquitination", "protein modification by small protein conjugation", "ubiquitin-like protein transferase activity", "ubiquitin activating enzyme activity", "ubiquitin-protein transferase activity" and "ubiquitin conjugating enzyme activity" were also significantly enriched (Fig. 6A and Table S6). KEGG enrichment analysis results showed that 177 FaUBC genes were significantly enriched to 8 KEGG pathways ( $p$-value $<0.05)$ (Fig. $6 \mathrm{~B}$ and Table S7). Among them, 155 and 149 FaUBC genes were enriched into "Ubiquitin system" and "Ubiquitin mediated proteolysis" pathways, respectively. These annotations suggested that $F a U B C$ genes are involved in a variety of biological processes.

\section{Protein-protein interaction network prediction of FaUBC proteins}

To predict the molecular interactions of FaUBC proteins, a protein-protein interactional analysis was performed based on their orthologues AtUBC proteins in Arabidopsis. Of the 191 FaUBC proteins, 176 (92.1\%) displayed homology to 28 AtUBC proteins from Arabidopsis (Table S8). As illustrated in Fig. 7, SCE1 (homolog of FaUBC5/10/16/41) showed interactions with 19 proteins, including several UBC proteins (such as UBC27 and UEVID-4) and other proteins in strawberry like RING$\mathrm{H} 2$ protein (RBX1), SUMO-activating enzymes (SAE1B-1 and SAE2), and small ubiquitin-related modifier proteins (SUMO1, SUMO2 and SUMO3). It is also predicted that UBC27 (homolog of FaUBC115/123/126/133) can interact with 13 proteins. UEVID-4 (homolog of FaUBC56/76) also may interact directly with UBC proteins like UBC1, UBC13, UBC27, UBC35, and UBC36. In addition, RCE1 (homolog of FaUBC3/4/9/15/22/28/31/37/39/43/45/4 9) showed significant correlations with RBX1, SAE2 and CUL1 (Cullin protein).

\section{Transcript abundance analysis of FaUBC genes in strawberry}

To investigate the roles of FaUBC genes in the ripening of strawberry, the RNAseq-based expression was assessed. As illustrated in Fig. 8A, different FaUBC genes comprise different expression pattern during the fruit ripening process. Most genes were highly expressed in TR and FR stages. In general, according to their expression patterns, all the FaUBC genes could be clarified into 6 clusters as 


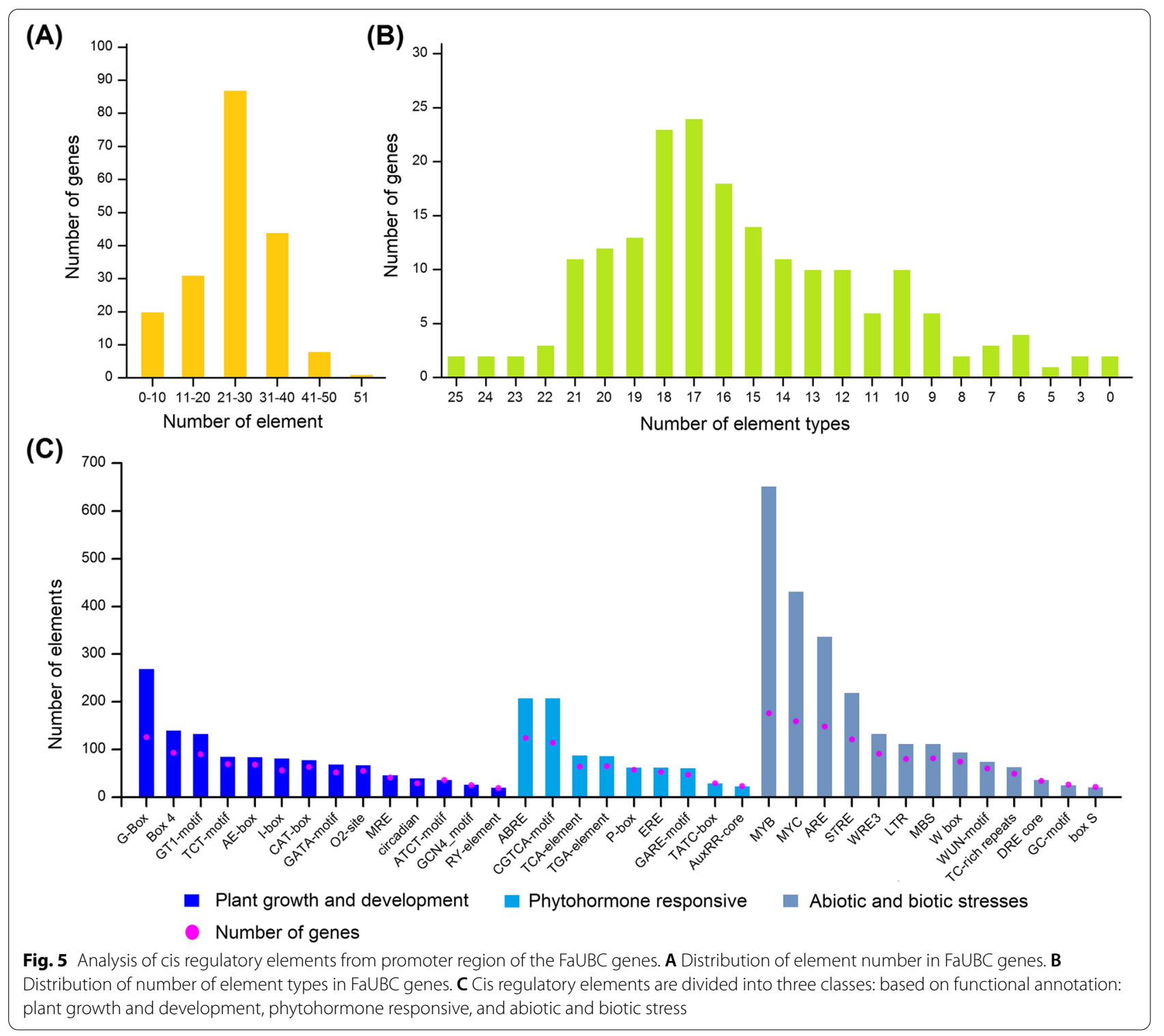

\section{(A)}

cellular macromolecule metabolic process primary metabolic process cellular protein metabolic process ligase activity
catalytic activity, acting on a protein protein ubiquitination protein modification process cellular metabolic process small molecule binding

anion binding
ligase activity, forming carbon-sulfur bonds
transferase activity transferase activity catalytic activity cellular protein modification process dification by small protein conjugation macromolecule metabolic process nitrogen compound metabolic process metabolic process bolic process
ion binding
(B)

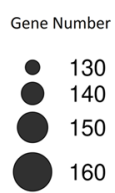

p-value

$4.44 \mathrm{e}-16$ 3.33e-16

$2.22 \mathrm{e}-16$

$1.11 \mathrm{e}-16$

$0.00 \mathrm{e}+00$

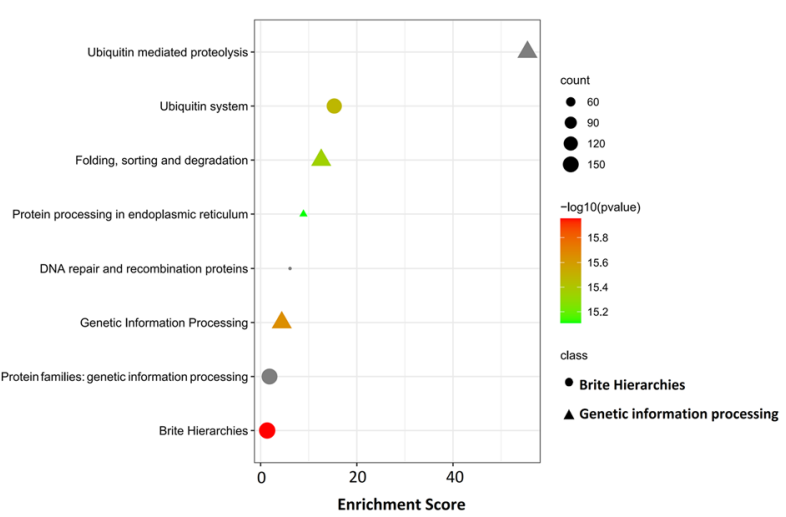

Fig. 6 Functional annotation of FaUBC genes. A The top 20 of $\mathrm{GO}$ enrichment function annotation. B KEGG pathway enrichment analysis 


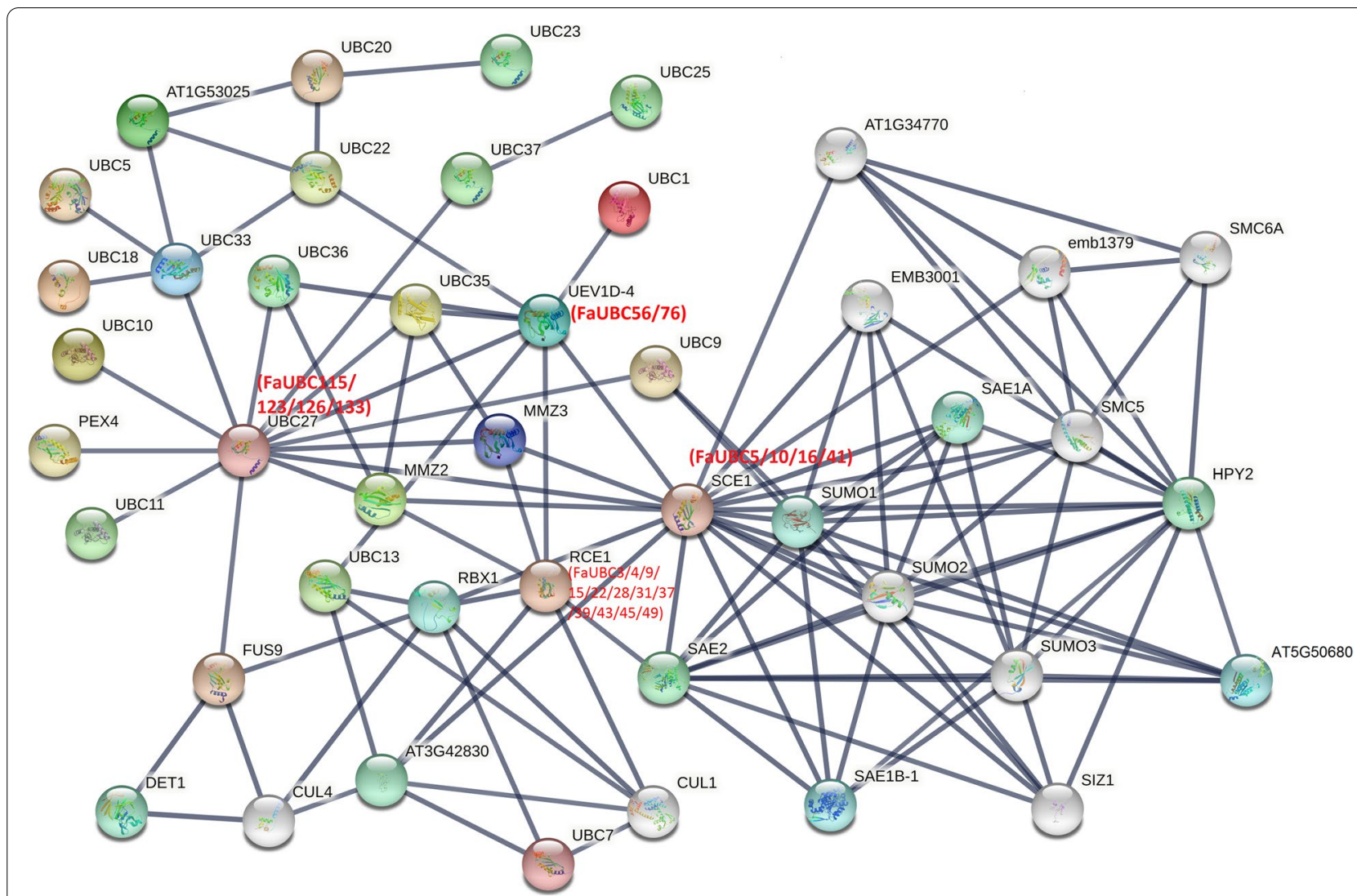

Fig. 7 Interaction network of FaUBC genes based on the orthologs in Arabidopsis. The interaction network is based on the Arabidopsis model. Details Annotation of associated Arabidopsis and strawberry UBC proteins are presented in Supplementary Table 7

shown in Fig. 8B. Among them, 14 genes belonged in cluster 2 showing a gradual increasing of expression during fruit ripening, and 41 members in cluster 3 exhibiting a sudden expression increase in the TR stage were speculated to be involved in fruit ripening. All the other genes involved in the other clusters were listed in Table S9.

\section{Validation of the function of $\mathrm{FaUBC76}$ and $\mathrm{FaUBC78}$ in strawberry fruit ripening}

To validate the roles of FaUBC genes in strawberry fruit ripening, two FaUBC genes (FaUBC76 and FaUBC78) included in the cluster 3 were selected for gene function analysis. A RT-qPCR analysis was performed to detect the UBC expression during fruit development stages. In agreement with the transcriptome data, the expression of FaUBC76 and FaUBC78 were abundantly expressed in FR, but at low levels in W stage (Figs. 9A and 10A). The two UBC genes were further validated by transient overexpression method.

At 5 days after injection, the fruit color of FaUBC76 overexpression group was significantly redder than the control group (Fig. 9B). A significant higher expression level in the FaUBC76 overexpression fruit than that of the control was observed, indicating the transient overexpression successfully resulted in the up-regulation of FaUBC76. The firmness of FaUBC76 overexpression fruit was lower than that of the control group (Fig. 9C), whereas the TA were not significant (Fig. 9D). In addition, the content of total anthocyanins in overexpression fruit was higher than that of the control group (Fig. 9E). Furthermore, RT-qPCR was used to measure the expression of a set of ripening-related genes, including ABA biosynthesis gene (NCED1), cell wall-related genes (cellulase $1 / 2, C E L 1 / 2$, and pectin lyase $1, P L 1$ ), anthocyanin biosynthesis related genes (chalcone isomerase, $\mathrm{CHI}$; anthocyanidin synthase, $A N S$ and $M Y B 10$ ). The results showed that FaMYB10, FaPL1, FaCEL1/2 were up-regulated, and FaNCED1, FaANS and FaCHI had no significant difference compared with that of the control fruit (Fig. 9E). These results indicated that FaUBC76 is a positive regulatory factor that promotes strawberry ripening.

Between CK and FaUBC78 overexpression fruit groups, the fruits were harvest after 8 days when the $C K$ turned to fully red first. The relative expression level of FaUBC78 was significantly increased in the overexpression fruit (Fig. 10B). Notably, the overexpression of 


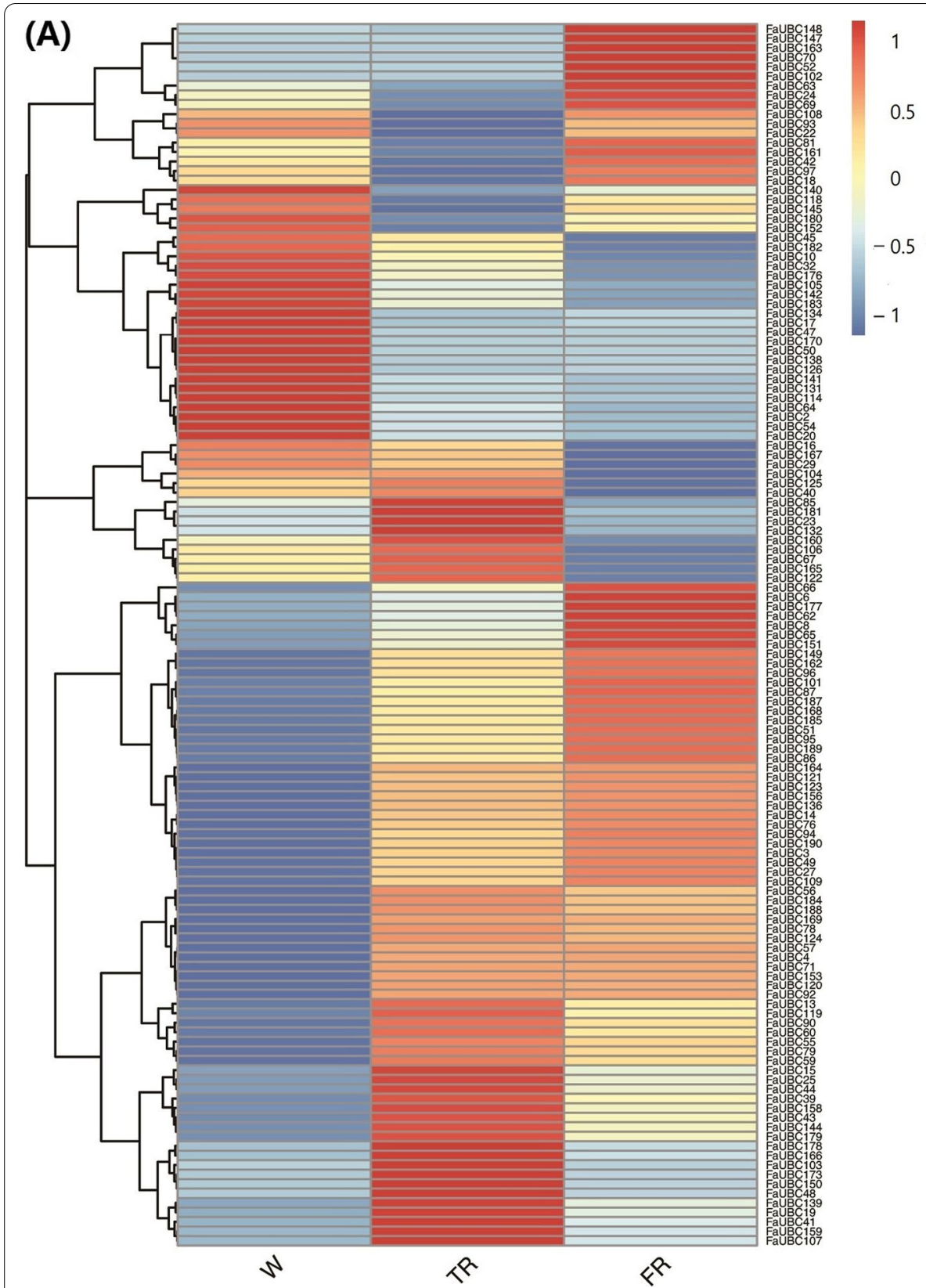

(B)
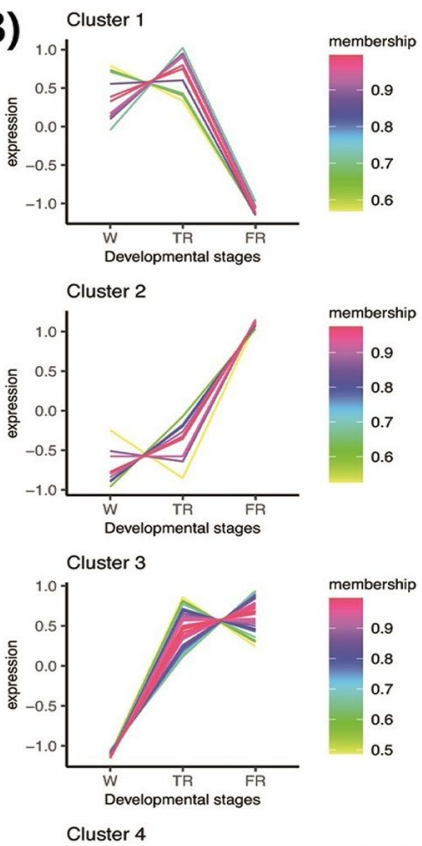

membership
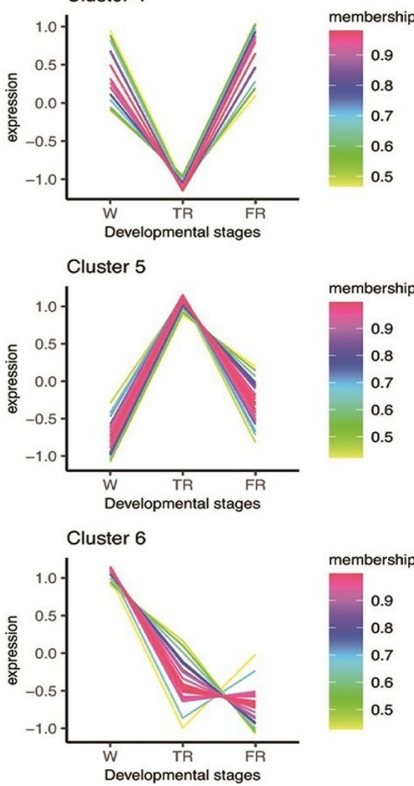

Fig. 8 Transcript abundance of FaUBC genes in different treatments. A Heatmap of gene transcript abundance based on transcriptome data. W: white; TR: turning red; FR: full red. $\mathbf{B}$ Clustering of gene expression trends

FaUBC78 significantly increased the firmness of strawberry fruit, but had no significant effects on fruit TA and anthocyanin content (Fig. 10C E). RT-qPCR was used to measure the transcript levels of a set of ripening-related genes, and the result showed that only the expression level of $\mathrm{FaCHI}$ was significant up-regulated in the FaUBC78 overexpressed fruit (Fig. 10F). These work reveals $F a U B C 78$ play a role in inhibiting the decrease of fruit firmness and delaying maturation time during fruit ripening.

\section{Discussion}

In this study, a total of 191 UBC genes were identified in the genome of Fragaria $\times$ ananassa. The number of FaUBC genes is far more than the 45, 48, 52, 75, and 74 UBC genes isolated from the genome of Arabidopsis [32], 

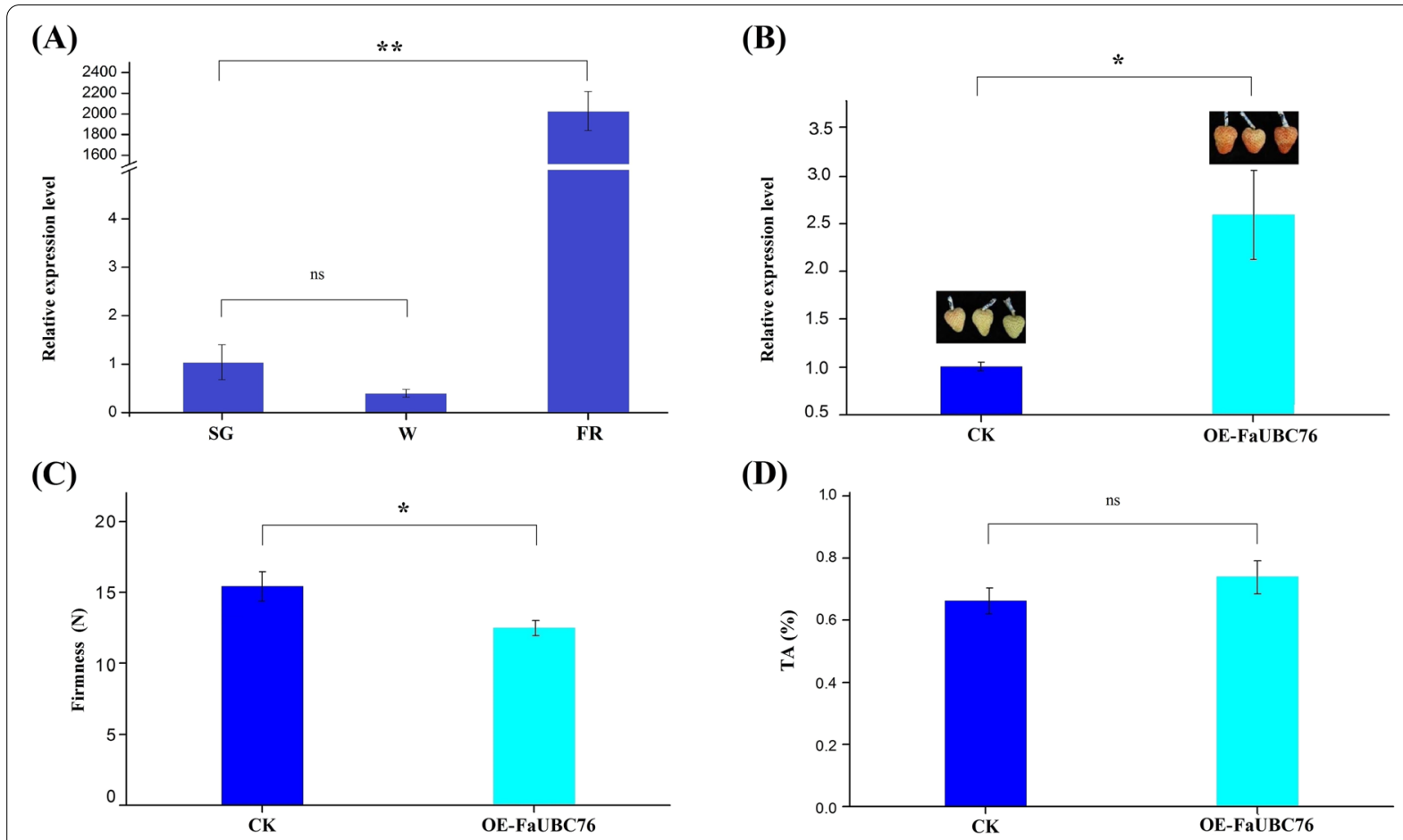

(E)

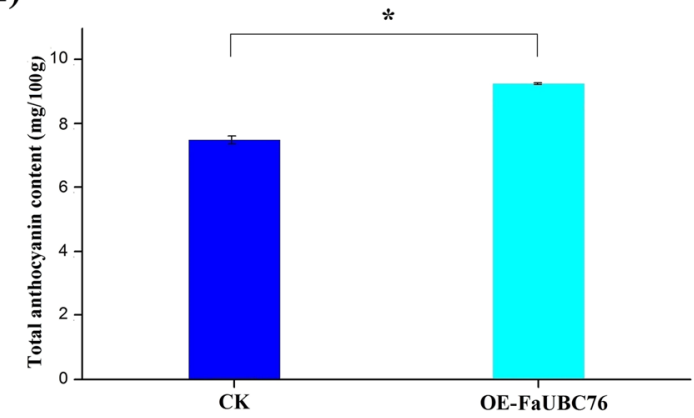

(F)

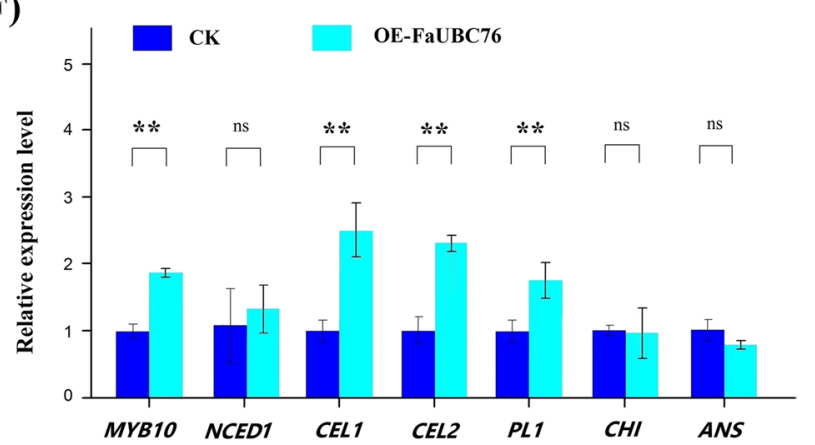

Fig. 9 Overexpression for the FaUBC76 gene in strawberry fruits. The injected fruits were harvested 5 days after injection. A RT-qPCR analysis of the relative expression level of FaUBC76 at small green (SG), white (W) and partial red (PR) stages. B Transcript levels of FaUBC76 in the control and overexpression fruit by RT-qPCR. C-E Fruit firmness, titratable acid content, anthocyanin content in the control and FaUBC76 overexpression fruit. F Transcript levels of ripening-related genes in the control and FaUBC76 overexpression fruits. Data are expressed as the mean \pm SD of three replicates, and each repeat included 10 fruit individuals. Asterisks indicate significant differences $\left({ }^{*} P<0.05,{ }^{*} P<0.01, \mathrm{~ns} P>0.05\right)$ between the means of the overexpression fruits and the control group

rice [5], tomato [6], banana [7] and maize [33], which indicates that the UBC family expanded in strawberry. Gene duplication events such as whole genome duplication, tandem duplication, segmental duplication, and transposition have a remarkable role in the expansion of gene family members in genomes [34], and increasing evidences has shown that whole genome duplication are largely responsible for the expansion of gene families in cultivated strawberry, such as the GST, GMP and RALF gene families [35-37]. Additionally, the genome sizes of Arabidopsis, strawberry, rice, tomato, maize, and banana are $\sim 125, \sim 240, \sim 466, \sim 466, \sim 900, \sim 2300$, and $523 \mathrm{Mb}$, respectively. Clearly, the higher number of UBC-encoding genes in strawberry can not only be explained by genome size.

Pear, peach, and strawberry are important fruit producing species belong to Rosaceae family, which share similarly genetic backgrounds. Previous studies also showed that there were more collinear regions in the genome, suggesting that the phylogenetic relationship of these three species was closely related [38]. In our study, a total of 69 and 74 UBC paralogous gene pairs 
(A)

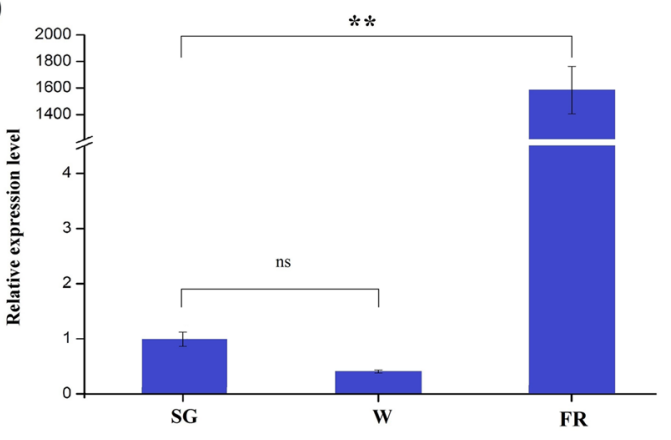

(C)

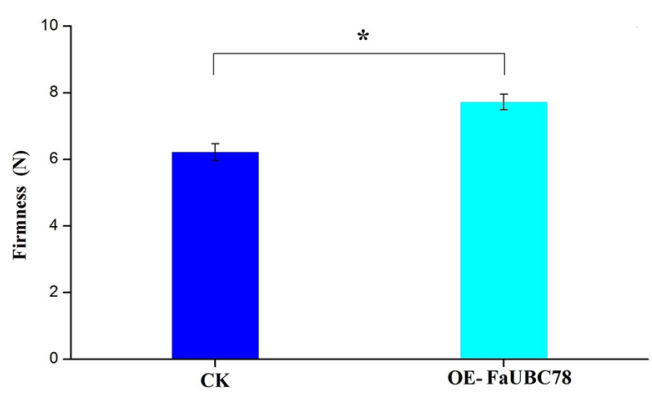

(E)

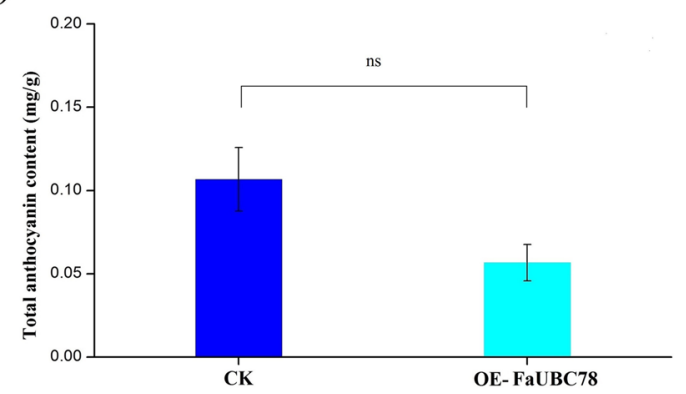

(B)

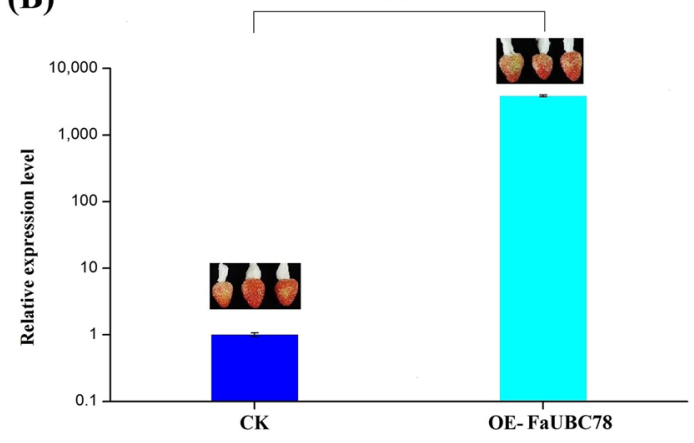

(D)

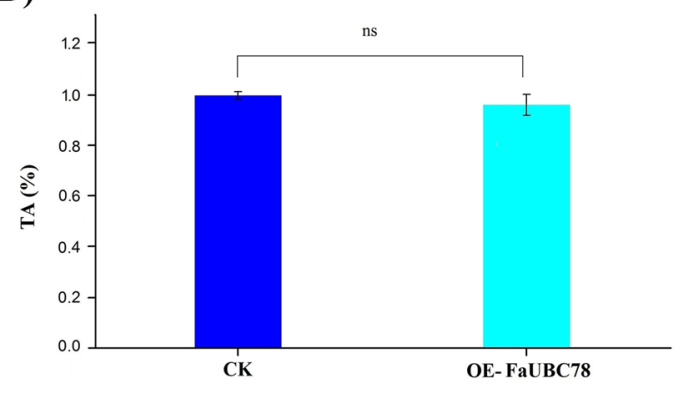

(F)

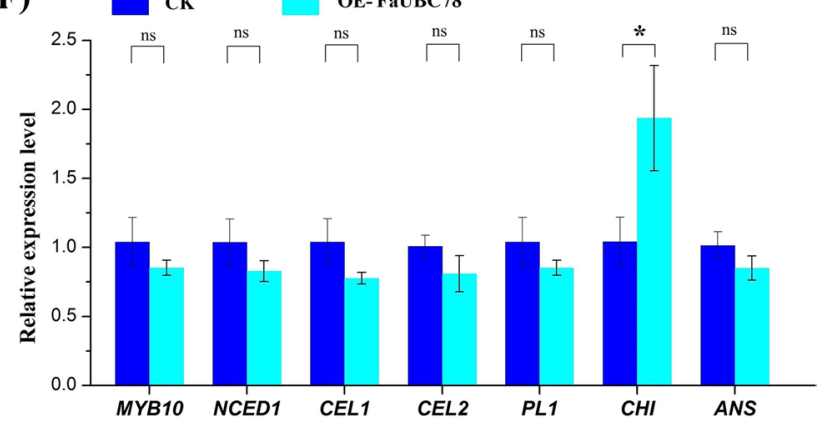

Fig. 10 Overexpression for the FaUBC78 gene in strawberry fruits. The injected fruits were harvested 8 days after injection. A RT-qPCR analysis of the relative expression level of FaUBC78 at small green (SG), white (W) and partial red (PR) stages. B Transcript levels of FaUBC78 in the control and overexpression fruit by RT-qPCR. C-E Fruit firmness, titratable acid content, anthocyanin content in the control and FaUBC78 overexpression fruit. F Transcript levels of ripening-related genes in the control and FaUBC78 overexpression fruits. Data are expressed as the mean \pm SD of three replicates, and each repeat included 10 fruit individuals. Asterisks indicate significant differences $\left({ }^{*} P<0.05,{ }^{*} P<0.01\right.$, ns $\left.P>0.05\right)$ between the means of the overexpression fruits and the control group

were revealed in strawberry/peach and strawberry/ pear, respectively, supporting a strong evolutionary relationship of UBC genes in Rosaceae species. The Ka/ Ks value can further explain the gene selection pressure and evolution rate positive selection: $\mathrm{Ka} / \mathrm{Ks}>1$ suggested positive selection, $\mathrm{Ka} / \mathrm{Ks}=1$ suggested neutral selection, and $\mathrm{Ka} / \mathrm{Ks}<1$ suggested negative selection [39]. All paralogous genes in UBC gene family among strawberry/peach and strawberry/pear have experienced purifying selection with the ratio of $\mathrm{Ka} / \mathrm{Ks}$ less than one. These findings of collinearity analysis indicate that the genes were retained in Rosaceae species after whole genome duplication event and were strongly retained by stabilizing selection [40].

To further understand the regulatory network of UBC proteins, an interaction network was built. Most UBC proteins exhibited interactions with multiple proteins including several UBC proteins, RBX1, SUMO. A RING-H2 Protein, RBX1, bind the ubiquitin-conjugating enzyme E2 and bring it into close proximity with the E3 substrate [41]. And RCE1 (homolog of FaUBC3/4/9/15/2 $2 / 28 / 31 / 37 / 39 / 43 / 45 / 49$ ) which is interacts directly with RBX1 in Arabidopsis and play important role in early development [42]. There are other studies showing that 
an E2 ubiquitin ligase UBC-9 mediates covalent attachment of small ubiquitin-related modifier (SUMO) [43, 44]. This provided a useful reference for in-depth understanding the molecular interactions of FaUBC proteins.

Despite the potential functional significance of UBC members, only a few UBC family members have been described in horticultural plants with fruits as product organs, such as tomato [6], banana [7], papaya [45], grape [46] and pear [47]. Among them, several studies have been demonstrated that E2s are involved in the fruit ripening process. SIUBC32, which is upregulated during tomato (Solanum lycopersicum) fruit ripening and downregulated in the rin mutant, plays an important role in the regulation of fruit ripening [6]. Some of MaUBC genes are up- and downregulated during different ripening stages in banana (Musa acuminata) [7]. Thirteen (CpUB $C 4 / 6 / 7 / 8 / 9 / 11 / 12 / 14 / 16 / 19 / 20 / 28 / 34)$ and two (CpUBC2 and $C p U B C 10$ ) of the $34 \mathrm{Cp} U B C$ genes in papaya (Carica papaya) were up- or downregulated during the progression of fruit ripening, respectively [45]. To investigate the roles of $F a U B C$ genes in the ripening of strawberry, the expression pattern of $F a U B C$ genes were analyzed during the fruit ripening process. The results showed that cluster 3 exhibiting a sudden expression increase in the turning red stage were speculated to be involved in fruit ripening. Subsequently, two FaUBC genes (FaUBC76 and FaUBC78) included in the cluster 3 were randomly selected for gene function analysis by transient overexpression method. The results showed that FaUBC76 overexpression gave rise to higher anthocyanin content and lower firmness than the control. It has been documented that the color, firmness and soluble solids of fruit are important signs of strawberry ripening. For most fruit trees, fruit ripening is usually characterized by fruit softening and color transformation, accompanied by complex physiological and biochemical changes and volatile metabolism to form unique quality [48]. Furthermore, the gene expression analysis showed that FaUBC76 overexpression can significantly increase gene expression of FaMYB10, FaPL1, FaNCED1 and FaCEL1/2 that involved in strawberry ripening. Therefore, we speculate that FaUBC76 has a positive effect on the fruit development and ripening in strawberry. FaUBC78 overexpression significantly enhance the strawberry firmness, but had no significant effects on fruit TA and anthocyanin content. Moreover, only the expression level of $\mathrm{FaCHI}$ was significant up-regulated in the FaUBC78 overexpressed fruit, while other ripening-related genes had no significant change. Taken together, these findings suggested that the strawberry UBC family genes might be participated in the regulation of fruit development and ripening processes. However, only two genes of UBC family was studied in this experiment, so the regulation of other genes of the same family in strawberry fruit ripening needs to be further explored.

\section{Materials and methods}

Identification and comprehensive analysis of FaUBC genes

The UBC domain (PF00179) obtained from Pfam database was used as a query to search against the genome of strawberry downloaded from Genome Database for Rosaceae (GDR, https://www.rosaceae.org) [49]. The sequences with high score in the results were retrieved as putative UBC proteins. The number of amino acids, ORF length, putative protein molecular weights (MW) and isoelectric points (pI) for each sequence were obtained using a perl script. Multiple alignment was conducted using Geneious Prime software (https://www.genei ous.com/). The conserved domains in the FaUBC proteins were screened and annotated based on Pfam [50], and NCBI-CDD [51]. The exon-intron structure of the FaUBC genes was analyzed using Gene Structure Display Server v.2.0 (http://gsds.cbi.pku.edu.cn/index.php). To identify the conserved motifs of FaUBC proteins, the MEME online program (http://meme-suite.org/tools/ meme) was used with the following parameters: any number of repetition, maximum number of motifs was set as 10, and optimum motif length was set to 6-100 residues. The upstream $1500 \mathrm{bp}$ regions of FaUBC genes were downloaded from the genome data and were performed to analyze the cis regulatory element using PlantCARE (http://bioinformatics.psb.ugent.be/webtools/ plantcare/html/). The interaction networks of the identified proteins were analyzed by STRING (https://stringdb.org/). GO and KEGG functional annotation of FaUBC proteins were performed by PANNZER2 (http://ekhid na2.biocenter.helsinki.fi/sanspanz/) and DAVID (https:// david.ncifcrf.gov/), respectively.

\section{Phylogenetic and evolutionary analysis of UBC proteins}

The Arabidopsis UBC protein sequences were downloaded from the Arabidopsis information source (TAIR) database, and the genomic data for pear (Pyrus communis) and peach (Prunus persica) were downloaded from Genome Database for Rosaceae (GDR, https://www. rosaceae.org). Phylogenetic tree of FaUBC proteins was constructed using Clustal X v.2.0 and MEGA v.7.0 software [52] with the neighbor-joining (NJ) method, and a bootstrap test with 1000 replicates were chose to evaluate the support of interior branches. The collinear gene pair was calculated using MCScanX software (http://chibba. pgml.uga.edu/mcscan $2 /$ ), the synteny relationship was visualized by Circos (http://circos.ca/). Synonymous (Ks) and non-synonymous (Ka) substitutions per site between duplicated FaUBC gene pairs were subsequently calculated using KaKs Calculator v.1.2 software [53]. 
Plant materials, RNA extraction, and cDNA synthesis The strawberry (Fragaria $\times$ ananassa cv. Benihoppe) used as the experimental materials in our study were supplied by the Hanyuan County Dr. Luo Agriculture Co., Ltd. (Sichuan, China). The fruits at small green (SG), white (W) and full red (FR) stages were collected separately. These materials were quickly frozen and stored at $-80^{\circ} \mathrm{C}$ for subsequent experiments.

Total RNA was extracted from frozen strawberry fruit using the total RNA kit (Tiangen, Beijing, China) according to the manufacturer's instructions. The RNA concentration was determined using a NanoDrop ND 2000 spectrophotometer, and purity was measured by the ratio OD260/OD280 (1.8-2.0) and OD260/ OD230 (2.0-2.2). Finally, the integrity of the RNA was examined by $1.0 \%$ agarose gel electrophoresis. cDNA was synthesized from $1.0 \mu \mathrm{g}$ of total RNA using the TransGene reverse transcription kits (Beijing, China). All cDNA samples were diluted 1:10 with RNase-free water for gene cloning and quantitative real-time PCR (RT-qPCR).

\section{Transcript abundance analysis and RT-qPCR analysis of FaUBC genes}

The transcript abundance levels of FaUBC genes in strawberry were retrieved from the online transcriptomic data (SRA accession: SRX6381727). The expression cluster analysis was analyzed by TCseq $R$ package.

RT-qPCR analysis was carried out using SYBR Green Premix Ex TaqTM (Takara, Japan) on a CFX96 qPCR system (Bio-Rad, USA) in triplicate of each sample. The relative expression was calculated using the $2^{-\Delta \Delta \mathrm{Ct}}$ method [54]. Fa26S rRNA (accession: X58118) was used as the reference gene to standardize the raw data. All primers used in the present study were listed in Table S10.

\section{Transient overexpression of FaUBC76 and FaUBC78 in strawberry fruit}

Full-length coding sequence of FaUBC76 and FaUBC78 was amplified using the cDNA sample. Then these two genes were cloned into the modified PCAMBIA1301 vector with CaMV35s promoter, respectively. The recombinant vectors was then introduced into the Agrobacterium tumefaciens strain GV3101. Agrobacterium infiltration was performed based on the previously described method [55]. The agrobacterium suspension was injected in the entire fruits at white (W) stage when they were still attached to the plants by a sterile $1 \mathrm{~mL}$ syringe. The injected fruits were harvested when they turned fully red after injection. As control, fruits at the same stage were injected with bacteria containing empty vector. Each treatment included three replicates, and each repeat included 10 fruit individuals.

\section{Determination of the TSS, firmness, the TA and total anthocyanin content}

The fruit firmness was measured by using a digital display firmness tester (FR-5105, LUTRON, China). The total titratable acid (TA) content was measured by titration with $0.1 \mathrm{M} \mathrm{NaOH}$ titration, and expressed as a citric acid content percentage. Anthocyanin content determination was carried out by the $\mathrm{pH}$-differential method using buffer solutions of sodium acetate $(0.4 \mathrm{M}, \mathrm{pH} 1.0)$ and potassium chloride $(0.025 \mathrm{M}, \mathrm{pH} 4.5)$, and the absorbance was read at 520 and $700 \mathrm{~nm}$, respectively [56].

\section{Statistical analysis}

The experiment was arranged in a completely randomized design with three replications. Statistical analysis was performed using multivariate logistic regression with the IBM SPSS statistics program (SPSS Version 27). Data were represented as average $\pm \operatorname{STDEV}(n=3)$. Analysis of variance (ANOVA) was used to determine the significant difference when $p<0.05$.

\section{Supplementary Information}

The online version contains supplementary material available at https://doi. org/10.1186/s12870-021-03421-8.

Additional file 1: Figure S1. Schematic gene structure and motif location of FaUBC genes.

Additional file 2: Table S1. The basic information of FaUBC genes in the genome of cultivated strawberry.

Additional file 3: Table S2. Collinear pairs identified between Arabidopsis and strawberry.

Additional file 4: Table S3. Ka, Ks and $\mathrm{Ka} / \mathrm{Ks}$ of paralogous gene pairs of FaUBC genes.

Additional file 5: Table S4. Ka, Ks and Ka/Ks of paralogous gene pairs of UBC genes among three Rosaceae species.

Additional file 6: Table S5. Analysis of cis-regulatory elements in the promoter regions of FaUBC genes.

Additional file 7: Table S6. GO categories and distribution of FaUBC genes.

Additional file 8: Table S7. KEGG function classification of FaUBC genes.

Additional file 9: Table S8. The AtUBC proteins in protein-protein interaction network and the homologous FaUBC proteins in strawberry.

Additional file 10: Table S9. Expression of FaUBC genes and expression clusters.

Additional file 11: Table S10. Primers of ripening-related genes used to detect the expression level in overexpressed fruits.

Acknowledgements

Not Applicable. 


\section{Authors' contributions}

M.Y.L., L.X.W., Y.T.L. and Y.L. (Ya Luo) initiated and designed the research. L.X.W., Y.T.L., Q.C., and Y.T.Z. performed the experiments. Y.X.L., Y.L. (Yu Long), Y.W., C.Y.L. and Y.Z. analyzed the data. M.Y.L. and L.X.W. wrote the paper. H.R.T., P.W.C. and X.R.W. revised the paper. Y.L. (Ya Luo) contributed reagents/materials/analysis tools. All authors have read and approved the manuscript.

\section{Funding}

This work was supported by the National Natural Science Foundation of China (3180817), the Key Projects of Sichuan Provincial Science and Technology Department (2018NZ0126), the Open Research Fund Program of Departmental and Municipal Coconstruction of Crops Genetic Improvement of Hill Land Key Laboratory of Sichuan Province (2021CGIHL05), the Coopperation Project of Ya'an City and Sichuan Agricultural University. The funders had no role in study design, data collection and analysis, decision to publish, or preparation of the manuscript.

\section{Availability of data and materials}

The following information was supplied regarding data availability: RNA-Seq data are available at the NCBI Sequence Read Archive: SRX6381727.

The genome sequence data is available at GDR database (GDR, https://www. rosaceae.org).

\section{Declarations}

\section{Ethics approval and consent to participate}

Not applicable.

\section{Consent for publication}

Not applicable.

\section{Competing interests}

The authors declare that they have no competing interests.

\section{Author details}

${ }^{1}$ College of Horticulture, Sichuan Agricultural University, Chengdu 611130, China. ${ }^{2}$ Institute of Pomology and Olericulture, Sichuan Agricultural University, Chengdu 611130, China. ${ }^{3}$ Departmental and Municipal Co-construction of Crops Genetic Improvement of Hill Land Key Laboratory of Sichuan, Nanchong 637000, China.

Received: 20 October 2021 Accepted: 24 December 2021 Published online: 19 January 2022

\section{References}

1. Santner A, Estelle M. The ubiquitin-proteasome system regulates plant hormone signaling. Plant J. 2010;61:1029-40. https://doi.org/10.1111/j. 1365-313X.2010.04112.x.

2. Hershko A, Ciechanover A. The ubiquitin system for protein degradation. Annu Rev Biochem. 1992;61:761-807. https://doi.org/10.1146/annurev.bi. 61.070192 .003553 .

3. Lee JH, Kim WT. Regulation of abiotic stress signal transduction by E3 ubiquitin ligases in Arabidopsis. Mol Cells. 2011;3:201-8. https://doi.org/ 10.1007/s10059-011-0031-9.

4. Sharma B, Joshi D, Yadav PK, Gupta AK, Bhatt TK. Role of ubiquitin-mediated degradation system in plant biology. Front Plant Sci. 2016;7:806. https://doi.org/10.3389/fpls.2016.00806.

5. Bae H, Kim WT. Classification and interaction modes of 40 rice E2 ubiquitin-conjugating enzymes with 17 rice ARM-U-box E3 ubiquitin ligases. Biochem Biophys Res Commun. 2014;444(4):575-80. https://doi. org/10.1016/j.bbrc.2014.01.098.

6. Wang Y, Wang W, Cai J, Zhang Y, Qin G, Tian S. Tomato nuclear proteome reveals the involvement of specific E2 ubiquitin-conjugating enzymes infruit ripening. Genome Biol. 2014;15:548. https://doi.org/10.1186/ s13059-014-0548-2.

7. Dong $\mathrm{C}$, Hu H, Jue D, Zhao Q, Chen $\mathrm{H}$, Xie J, et al. The banana E2 gene family: genomic identification, characterization, expression profiling analysis. Plant Sci. 2016;245:11-24. https://doi.org/10.1016/j.plantsci.2016. 01.003.

8. Liu WG, Tang X, Zhu X, Qi XH, Zhang N, Si HJ. Genome-wide identification and expression analysis of the E2 gene family in potato. Mol Biol Rep. 2019:46(1):777-91. https://doi.org/10.1007/s11033-018-4533-9.

9. van Wijk SJ, Timmers HT. The family of ubiquitin-conjugating enzymes (e2s): deciding between life and death of proteins. FASEB J. 2010;24(4):981-93. https://doi.org/10.1096/fj.09-136259.

10. Ahn MY, Oh TR, Seo DH, Kim JH, Cho NH, Kim WT. Arabidopsis group XIV ubiquitin-conjugating enzymes AtUBC32, AtUBC33, and AtUBC34 play negative roles in drought stress response. J Plant Physiol. 2018;230:73-9. https://doi.org/10.1016/j.jplph.2018.08.010.

11. Jue $D$, Sang $X$, Liu L, Shu B, Wang Y, Xie J, et al. The ubiquitin-conjugating enzyme gene family in Longan (Dimocarpus longan Lour.): genome-wide identification and gene expression during flower induction and abiotic stress responses. Molecules. 2018;23(3):662. https://doi.org/10.3390/ molecules23030662.

12. Zhou B, Mural RV, Chen X, Oates ME, Connor RA, Martin GB, et al. A subset of ubiquitin-conjugating enzymes is essential for plant immunity. Plant Physiol. 2017;173(2):1371-90. https://doi.org/10.1104/pp.16.01190.

13. Qin B. The function of Rad6 gene in Hevea brasiliensis extends beyond DNA repair. Plant Physiol Biochem. 2013;66:134-40. https://doi.org/10. 1016/j.plaphy.2013.02.013.

14. Tsui C, Raguraj A, Pickart CM. Ubiquitin binding site of the ubiquitin E2 variant (UEV) protein Mms2 is required for DNA damage tolerance in the yeast RAD6 pathway. J Biol Chem. 2005;280(20):19829-35. https://doi. org/10.1074/jbc.M414060200.

15. Cui F, Liu L, Zhao Q, Zhang Z, Li Q, Lin B, et al. Arabidopsis ubiquitin conjugase UBC32 is an ERAD component that functions in brassinosteroidmediated salt stress tolerance. Plant Cell. 2012;24(1):233-44. https://doi. org/10.1105/tpc.111.093062.

16. Xu L, Ménard R, Berr A, Fuchs J, Cognat V, Meyer D, et al. The E2 ubiquitinconjugating enzymes, AtUBC1 and AtUBC2, play redundant roles and are involved in activation of FLC expression and repression of flowering in Arabidopsis thaliana. Plant J. 2009;57:279-88. https://doi.org/10.1111/j. 1365-313X.2008.03684.x.

17. Wen R, Newton L, Li G, Wang H, Xiao W. Arabidopsis thaliana UBC13: implication of error-free DNA damage tolerance and Lys63-linked polyubiquitylation in plants. Plant Mol Biol. 2006;61:241-53. https://doi.org/ 10.1007/s11103-006-0007-x.

18. Li W, Schmidt W. A lysine-63-linked ubiquitin chain-forming conjugase, UBC13, promotes the developmental responses to iron deficiency in Arabidopsis roots. Plant J. 2010;62:330-43. https://doi.org/10.1111/j.1365313X.2010.04150.X.

19. Mural RV, Liu Y, Rosebrock TR, Brady JJ, Hamera S, Connor RA, et al. The tomato Fni3 lysine-63-specific ubiquitin-conjugating enzyme and suv ubiquitin E2 variant positively regulate plant immunity. Plant Cell. 2013;25:3615-31. https://doi.org/10.1105/tpc.113.117093.

20. Baloglu MC, Patir MG. Molecular characterization, 3D model analysis, and expression pattern of the CmUBC gene encoding the melon ubiquitinconjugating enzyme under drought and salt stress conditions. Biochem Genet. 2014;52(1-2):90-105. https://doi.org/10.1007/s10528-013-9630-9.

21. Millyard L, Lee J, Zhang C, Yates G, Sadanandom A. The ubiquitin conjugating enzyme, Ta U4 regulates wheat defence against the phytopathogen Zymoseptoria tritici. Sci Rep. 2016;6:35683. https://doi.org/10.1038/ srep35683.

22. Wang $\mathrm{S}$, Cao L, Wang $\mathrm{H}$. Arabidopsis ubiquitin-conjugating enzyme UBC22 is required for female gametophyte development and likely involved in Lys1 1-linked ubiquitination. J Exp Bot. 2016;67(11):3277-88. https://doi.org/10.1093/jxb/erw142.

23. Li C, Jia H, Chai Y, Shen Y. Abscisic acid perception and signaling transduction in strawberry: a model for non-climacteric fruit ripening. Plant Signal Behav. 2011;6(12):1950-3. https://doi.org/10.4161/psb.6.12.18024.

24. Mezzetti B, Landi L, Pandolfini T, Spena A. The defH9-iaaM auxin-synthesizing gene increases plant fecundity and fruit production in strawberry and raspberry. BMC Biotechnol. 2004:4:4. https://doi.org/10.1186/ 1472-6750-4-4

25. Narayanan KR, Mudge KW, Poovaiah BW. Demonstration of auxin binding to strawberry fruit membranes. Plant Physiol. 1981;68(6):1289-93. https:// doi.org/10.1104/pp.68.6.1289. 
26. Jia HF, Chai YM, Li CL, Lu D, Luo JJ, Qin L, et al. Abscisic acid plays an important role in the regulation of strawberry fruit ripening. Plant Physiol. 2011;157(1):188-99. https://doi.org/10.1104/pp.111.177311.

27. Stone SL, Williams LA, Farmer LM, Vierstra RD, Callis J. KEEP ON GOING, a RING E3 ligase essential for Arabidopsis growth and development, is involved in abscisic acid signaling. Plant Cell. 2006;18(12):3415-28. https://doi.org/10.1105/tpc.106.046532.

28. Guo J, Wang S, Yu X, Dong R, Li Y, Mei X, et al. Polyamines regulate strawberry fruit ripening by $A B A, I A A$, and ethylene. Plant Physiol. 2018;177(1):339-51. https://doi.org/10.1104/pp.18.00245.

29. Jia H, Jiu S, Zhang C, Wang C, Tariq P, Liu Z, et al. Abscisic acid and sucrose regulate tomato and strawberry fruit ripening through the abscisic acidstress-ripening transcription factor. Plant Biotechnol J. 2016;14(10):204565. https://doi.org/10.1111/pbi.12563.

30. Sutter JU, Sieben C, Hartel A, Eisenach C, Thiel G, Blatt MR. Abscisic acid triggers the endocytosis of the Arabidopsis KAT1 K+ channel and its recycling to the plasma membrane. Curr Biol. 2007;17(16):1396-402. https://doi.org/10.1016/j.cub.2007.07.020.

31. Chen YT, Liu H, Stone S, Callis J. ABA and the ubiquitin E3 ligase KEEP ON GOING affect proteolysis of the Arabidopsis thaliana transcription factors ABF1 and ABF3. Plant J. 2013;75(6):965-76. https://doi.org/10.1111/tpj. 12259.

32. Kraft E, Stone SL, Ma L, Su N, Gao Y, Lau OS, et al. Genome analysis and functional characterization of the E2 and RING-type E3 ligase ubiquitination enzymes of Arabidopsis. Plant Physiol. 2005;139(4):1597-611. https:// doi.org/10.1104/pp.105.067983.

33. Jue D, Sang X, Lu S, Dong C, Zhao Q, Chen H, et al. Genome-wide identification, phylogenetic and expression analyses of the ubiquitin-conjugating enzyme gene family in maize. PLoS One. 2015;10(11):e0143488. https://doi.org/10.1371/journal.pone.0143488.

34. Islam MS, Choudhury M, Majlish AN, Islam T, Ghosh A. Comprehensive genome-wide analysis of glutathione S-transferase gene family in potato (Solanum tuberosum L.) and their expression profiling in various anatomical tissues and perturbation conditions. Gene. 2018;639:149-62. https:// doi.org/10.1016/j.gene.2017.10.007.

35. Lin Y, Zhang L, Zhang J, Zhang Y, Wang Y, Chen Q, et al. Identification of anthocyanins-related glutathione S-transferase (GST) genes in the genome of cultivated strawberry ( Fragaria $\times$ ananassa). Int J Mol Sci. 2020;21(22):8708. https://doi.org/10.3390/ijms21228708.

36. Lin Y, Zhang J, Wu L, Zhang Y, Chen Q, Li M, et al. Genome-wide identification of GMP genes in Rosaceae and functional characterization of FaGMP4 in strawberry (Fragaria x ananassa). Genes Genomics. 2021;43(6):587-99. https://doi.org/10.1007/s13258-021-01062-7.

37. Zhang $H$, Jing $X$, Chen $Y$, Liu Z, Xin Y, Qiao $Y$. The genome-wide analysis of RALF-like genes in strawberry (wild and cultivated) and five other plant species (Rosaceae). Genes. 2020;11(2):174. https://doi.org/10.3390/genes 11020174.

38. Li Q, Qiao X, Yin H, Zhou Y, Dong H, Qi K, et al. Unbiased subgenome evolution following a recent whole-genome duplication in pear (Pyrus bretschneideri Rehd.). Hortic Res. 2019;6:34. https://doi.org/10.1038/ s41438-018-0110-6.

39. Hurst LD. The Ka/Ks ratio: diagnosing the form of sequence evolution. Trends Genet. 2002;18(9):486-7. https://doi.org/10.1016/s0168-9525(02 02722-1.

40. Lynch M, Conery JS. The evolutionary fate and consequences of duplicate genes. Science. 2000;290(5494):1151-5. https://doi.org/10.1126/science. 290.5494.1151.

41. Gray WM, Hellmann H, Dharmasiri S, Estelle M. Role of the Arabidopsis RING-H2 protein RBX1 in RUB modification and SCF function. Plant Cell. 2002;14(9):2137-44. https://doi.org/10.1105/tpc.003178.

42. Dharmasiri S, Dharmasiri N, Hellmann H, Estelle M. The RUB/Nedd8 conjugation pathway is required for early development in Arabidopsis. EMBO J. 2003;22(8):1762-70. https://doi.org/10.1093/emboj/cdg190.

43. Huang X, Tao Y, Gao J, Zhou X, Tang S, Deng C, et al. UBC9 coordinates inflammation affecting development of bladder cancer. Sci Rep. 2020;10(1):20670. https://doi.org/10.1038/s41598-020-77623-9.

44. Boulton SJ, Gartner A, Reboul J, Vaglio P, Dyson N, Hill DE, et al. Combined functional genomic maps of the C. elegans DNA damage response. Science. 2002;295(5552):127-31. https://doi.org/10.1126/science.1065986.

45. Jue D, Sang $X$, Shu B, Liu L, Wang Y, Jia Z, et al. Characterization and expression analysis of genes encoding ubiquitin conjugating domain-containing enzymes in Carica papaya. PLoS One. 2017;12(2):e0171357. https://doi.org/10.1371/journal.pone.0171357.

46. Gao Y, Wang Y, Xin H, Li S, Liang Z. Involvement of ubiquitin-conjugating enzyme (E2 gene family) in ripening process and response to cold and heat stress of Vitis vinifera. Sci Rep. 2017;7(1):13290. https://doi.org/10. 1038/s41598-017-13513-X.

47. Cao Y, Meng D, Chen Y, Abdullah M, Jin Q, Lin Y, et al. Comparative and expression analysis of ubiquitin conjugating domain-containing genes in two Pyrus species. Cells. 2018;7(7):77. https://doi.org/10.3390/cells70700 77.

48. Xie YG, Ma YY, Bi PP, Wei W, Liu J, Hu Y, et al. Transcription factor FvTCP9 promotes strawberry fruit ripening by regulating the biosynthesis of abscisic acid and anthocyanins. Plant Physiol Biochem. 2020;146:374-83. https://doi.org/10.1016/j.plaphy.2019.11.004.

49. Jung S, Lee T, Cheng CH, Buble K, Zheng P, Yu J, et al. 15 years of GDR: new data and functionality in the genome database for Rosaceae. Nucleic Acids Res. 2019;47(D1):D1137-45. https://doi.org/10.1093/nar/ gky1000.

50. Finn RD, Coggill P, Eberhardt RY, Eddy SR, Mistry J, Mitchell AL, et al. The Pfam protein families database: towards a more sustainable future. Nucleic Acids Res. 2016;44:D279-D85. https://doi.org/10.1093/nar/gkv13 44.

51. Marchler-Bauer A, Lu S, Anderson JB, Chitsaz F, Derbyshire MK, DeWeeseScott C, et al. CDD: a conserved domain database for the functional annotation of proteins. Nucleic Acids Res. 2011;39:D225-9. https://doi. org/10.1093/nar/gkq1189.

52. Kumar S, Stecher G, Tamura K. MEGA7: molecular evolutionary genetics analysis version 7.0 for bigger datasets. Mol Biol Evol. 2016;33(7):1870-4. https://doi.org/10.1093/molbev/msw054.

53. Zhang Z, Li J, Zhao XQ, Wang J, Wong GK, Yu J. KaKs_calculator: calculating $\mathrm{Ka}$ and $\mathrm{Ks}$ through model selection and model averaging. Genomics Proteomics Bioinformatics. 2006;4(4):259-63. https://doi.org/10.1016/ S1672-0229(07)60007-2.

54. Livak KJ, Schmittgen TD. Analysis of relative gene expression data using real-time quantitative PCR and the 2(-Delta Delta C(T)) method. Methods. 2001;25:402-8. https://doi.org/10.1006/meth.2001.1262.

55. Spolaore S, Trainotti, Casadoro G. A simple protocol for transient gene expression in ripe fleshy fruit mediated by Agrobacterium. J Exp Bot. 2001;52(357):845-50. https://doi.org/10.1093/jexbot/52.357.845.

56. Liu R, Zhang M, Li X. Comparisons of extraction solvents and quantitative methods of analysis of anthocyanins in strawberry and blueberry fruits. Acta Hortic Sin. 2008;35:655-60. https://doi.org/10.3724/SP.J.1005.2008. 01083.

\section{Publisher's Note}

Springer Nature remains neutral with regard to jurisdictional claims in published maps and institutional affiliations.

Ready to submit your research? Choose BMC and benefit from

- fast, convenient online submission

- thorough peer review by experienced researchers in your field

- rapid publication on acceptance

- support for research data, including large and complex data types

- gold Open Access which fosters wider collaboration and increased citations

- maximum visibility for your research: over $100 \mathrm{M}$ website views per year

At BMC, research is always in progress.

Learn more biomedcentral.com/submissions 